\title{
2 Recurrent Hierarchical Patterns and the Fractal Distribution of
}

\section{Fossil Localities}

$4 \quad$ Materials and Methods

Geographic coordinates (longitude and latitude) of terrestrial and marine fossil collections of

6 seventeen chronostratigraphic bins, from the conterminous United States were obtained from the

7 Paleobiology Database (https://paleobiodb.org) for all taxa (Tables 1 and DR1; Fig. DR1). Spatial

8 resolution of the collections varies widely, with most recorded at 0.1 degree resolution and some may

9 be incorrectly entered in the database (Peters and Heim 2010). In addition, collections from different

10 units within the same section may be given the same coordinates. I attempted to correct for these

11 issues by culling out collections with duplicate coordinates, producing unique "localities" for each

12 bin, and by using a 0.1 degree as the grain of the data in the lacunarity analyses. For the Lower and

13 Upper Cretaceous bins, localities resolved only to “Cretaceous” were removed. Cambrian,

14 Ordovician and Silurian terrestrial sites were sparse (Heim and Peters, 2011), and were not analyzed.

15 The majority of the analyses were conducted using PASSaGE v. 2.0 (Rosenberg and

16 Anderson, 2011), with cross-checks done within ESRI ArcMap ${ }^{\mathrm{TM}}$ and software written by the author.

17 Coordinates were converted to 0.1 degree resolution grids within PASSaGE; multiple sites

18 within each grid cell were aggregated to a total count. Lacunarity analysis (Plotnick et al., 1993;

19 Plotnick et al., 1996) was used to determine the deviation from random expectation at multiple scales

20 (for example, a pattern could be made of randomly spaced clumps of points) over $25 \%$ of the total

21 extent of the grid. In lacunarity analysis, a "gliding box" of linear size $l$ is placed over one corner of

22 the map and the total number of points in that box are counted. The box is then moved one unit,

23 overlapping the previous box and the count repeated. This is then repeated so that the map is 
24 exhaustively resampled. The dimensionless lacunarity $\wedge$ at that box size is then determined from the

25 mean $\bar{x}$ and variance $s^{2}$ of the number of points in the box:

$$
\Lambda=1+\frac{s^{2}}{\bar{x}^{2}}
$$

This is then repeated for a range of box sizes and $\log \Lambda$ is plotted against versus log box size $l$.

Higher values of $\Lambda$ at a given scale represent more clumping; that is, boxes tend to be either sparsely or densely occupied. Regular patterns, in contrast have low variance in occupancy and thus lower

31 lacunarity. If the points are csr, the entire resulting curve is concave upwards (Figure 1). A fractal

32 pattern, in contrast, yields a straight line with a negative slope (Plotnick et al., 1993). If the pattern is

33 a random fractal, some scatter around the line is expected. The y-intercept of the curves is a function

34 of the intensity at the lowest scale; sparse maps have higher lacunarities than dense maps of the same 35 size. Results for all analyses are in Fig. DR2 and DR3 with linear regressions shown for comparison 36 (see also Table 1).

As an independent test of csr, a matrix of interpoint distances for each bin was used to

calculate the Ripley’s $L(d)$ statistic. Spherical distances in kilometers among all points was determined for each bin and used to calculate the Ripley's $L(d)$ without edge correction over $10 \%$ of the span of the map. Edge correction produced no differences in the results. Ripley's L is a

41 modification of Ripley’s K second-order statistic (Haase, 1995; Young and Young, 1998; Fortin,

42 1999). In general, for a point pattern of area $A$ and number of points $n$, a circle of radius $d$ is drawn

43 around each occupied point $i$ and the number $I$ of other points within that radius is counted.

45 points per given circle area of radius $d$. Since this value will depend the density of points, it is then 46 corrected by the mean intensity $\lambda=\mathrm{n} / \mathrm{A}$. The resulting $\mathrm{K}(\mathrm{d})$ statistic (ignoring edge effects) is thus 47

$$
K(d)=\frac{A}{n^{2}} \sum_{i=1}^{n} I
$$


50 If we assume the points are Poisson distributed (complete spatial random) at the scale of resolution,

51 the expected number of points in an area of radius $d$ is $\pi \mathrm{d}^{2} \lambda$ (area $\mathbf{X}$ intensity) and

52 the corresponding value of $K(d)$ is $\pi d^{2} . L(d)$ is the deviation of $K(d)$ from $c s r$ :

$$
\mathrm{L}(\mathrm{d})=\mathrm{d}-\sqrt{\frac{K(d)}{\pi}}
$$

54 If, at the scale of resolution, the point pattern is $\operatorname{csr}, L(d)=0$. Patterns that are more regular, so that

55 there are fewer points within radius $d$ than the random expectation, have $\mathrm{L}(\mathrm{d})>0$. If the points are 56 more clumped that than csr, $\mathrm{L}(\mathrm{d})<0$. (Note that in some forms of $L(d), d$ is subtracted from $\sqrt{ } K(d) / \pi$, 57 so that the signs are reversed; here I use the version in PASSaGE). Values of L(d) are generated for a 58 range of radii and $L(d)$ is plotted against $d$.

59 All Ripley’s analyses resulted in clear rejection of csr. Results for six representative sets of 60 data are shown in Fig. DR4A; all of the curves are well-below the value of zero expected for a 61 random pattern. The same analysis for the random hierarchical map (Fig. 2D) is shown in Fig.

62 DR4B; the shape of the curve is essentially the same as for the data, supporting the interpretation of a 63 fractal pattern to the locality distribution. 
65 Figure DR1. Map of marine and terrestrial Miocene fossil localities from the lower 48 states.

66 Longitudes and latitudes downloaded from the Paleobiology Database (paleodb.org). Map drawn

67 using $\operatorname{ArcMap}^{\mathrm{TM}}$.

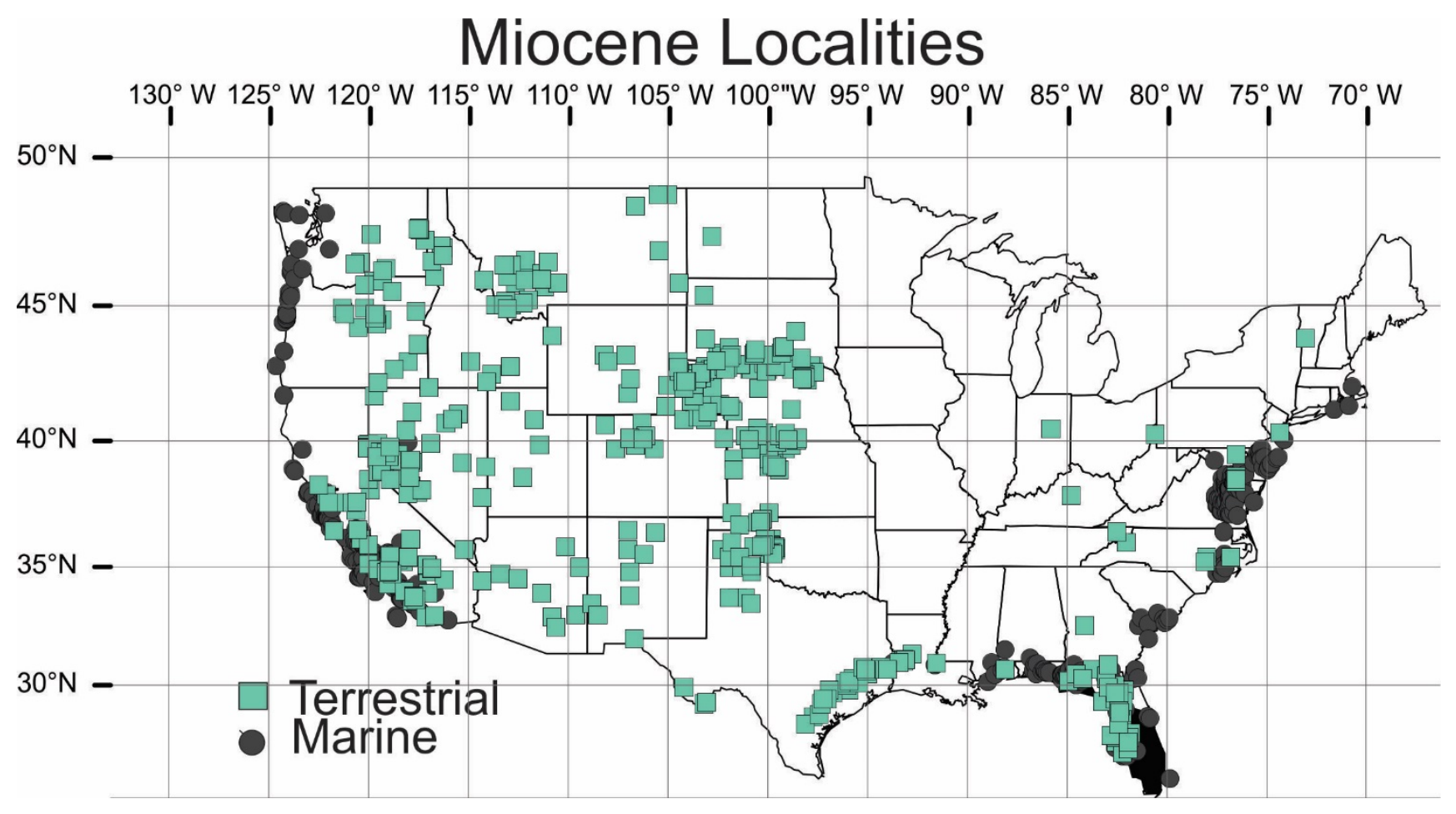


69 Figure DR2. Lacunarity plots for the marine sites in the seventeen bins. Lines are least-square

70 linear regressions. Box sizes are in units of $0.1^{\circ}$.

71
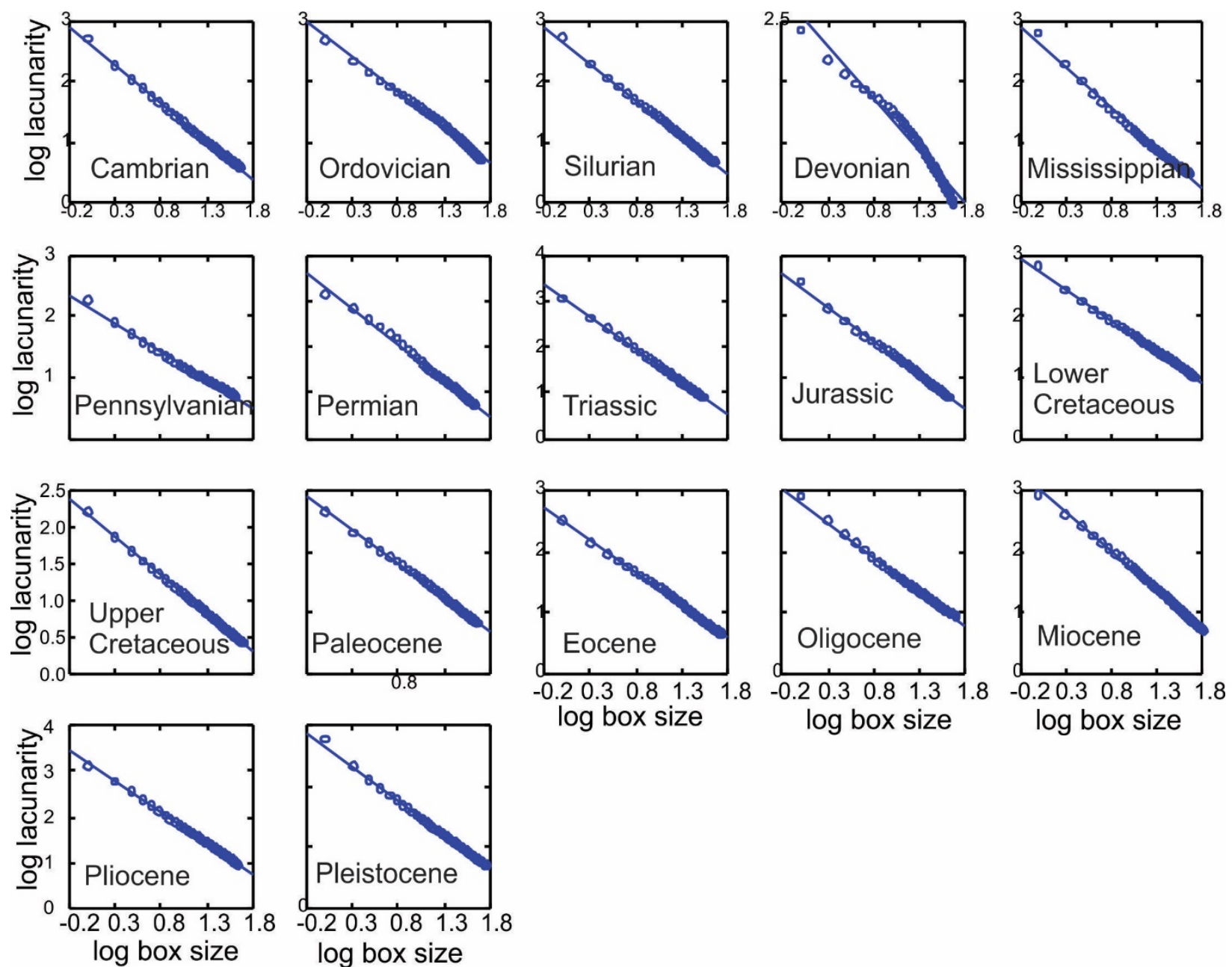

$\log$ box size 
74 Figure DR3. Lacunarity plots for the terrestrial sites in fourteen of the bins. There was

75 insufficient data for the Cambrian, Ordovician, and Silurian. Lines are least-square linear

76 regressions. Box sizes are in units of $0.1^{\circ}$.

77

78
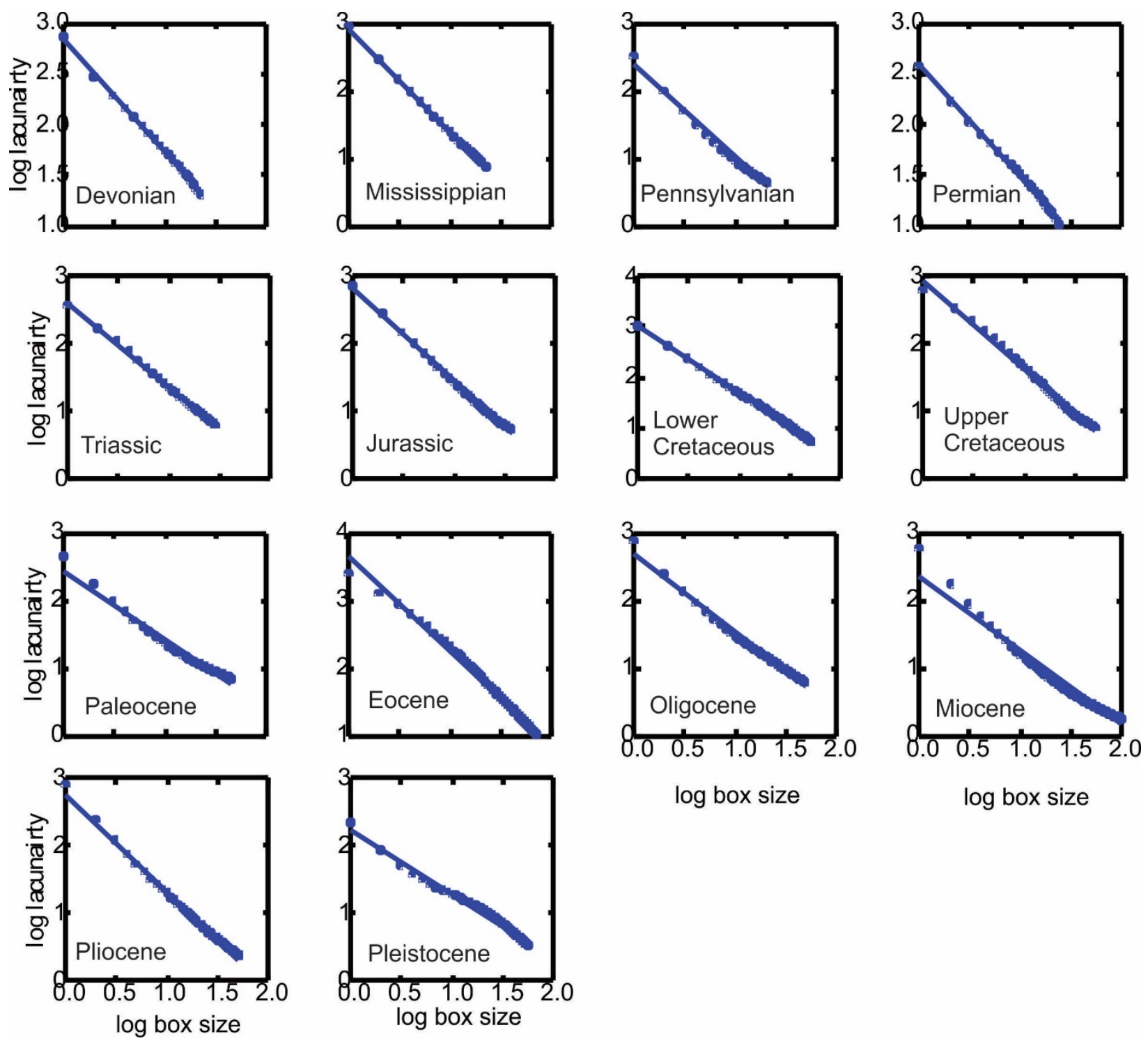

79

log box size 
81 Figure DR4. A) Ripley's L(d) plots for selected bins. A value of zero indicates complete spatial

82 randomness. B) Ripley's L(d) plot for the random multifractal map in Fig. 2C.

83
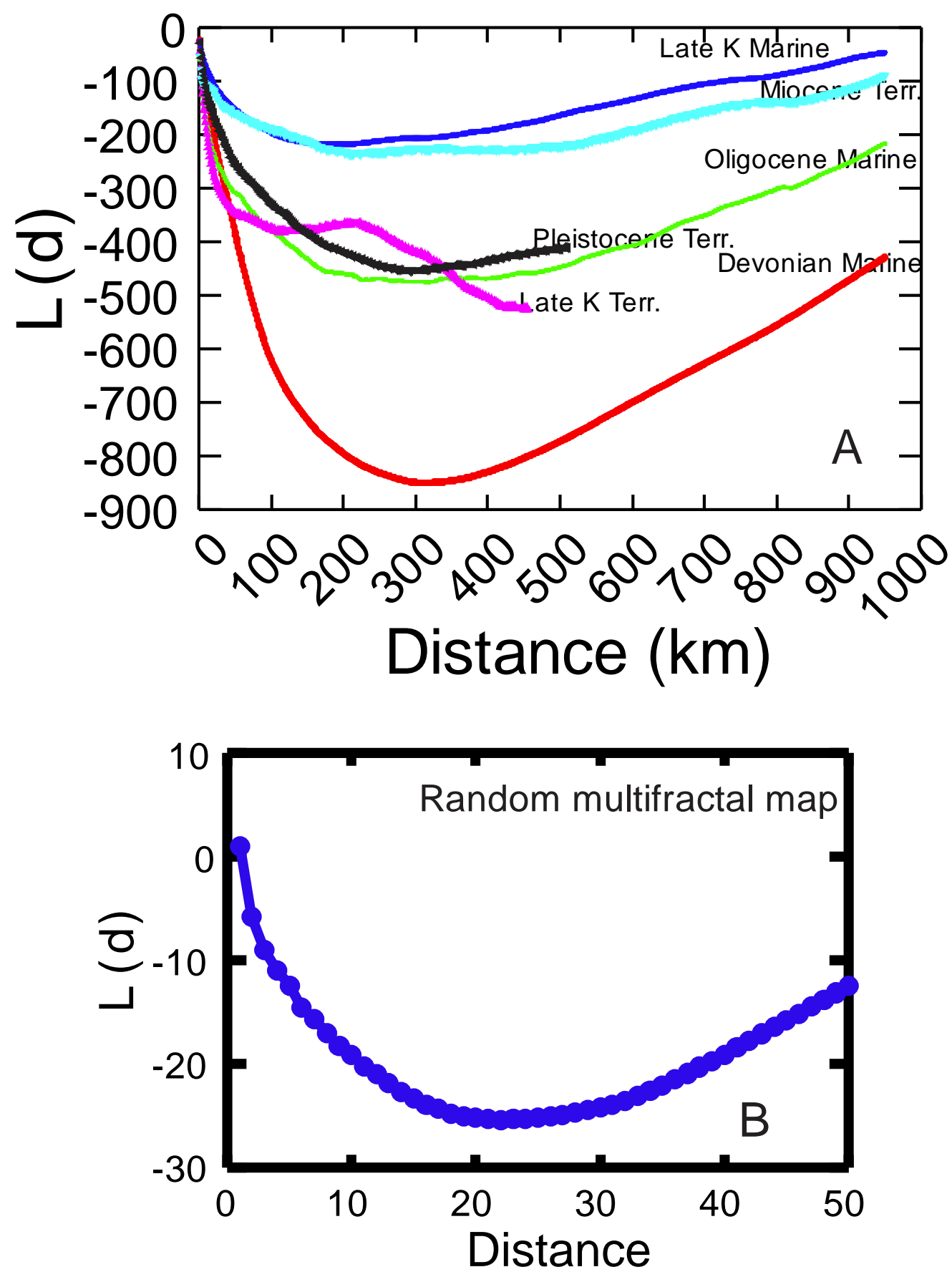
86 COLLECTION DOWNLOADS USED IN THIS PAPER.

\begin{tabular}{|c|c|}
\hline Marine & \\
\hline Cambrian & $\begin{array}{l}\text { paleobiodb.org/data1.2/colls/list.csv?datainfo\&rowcount\&interval=Cambrian,Cambrian\&cc=US } \\
\text { \&envtype=marine\&show=loc }\end{array}$ \\
\hline Ordovician & $\begin{array}{l}\text { paleobiodb.org/data1.2/colls/list.csv?datainfo\&rowcount\&interval=Ordovician,Ordovician\&cc= } \\
\text { US\&envtype=marine\&show=loc }\end{array}$ \\
\hline Silurian & $\begin{array}{l}\text { paleobiodb.org/data1.2/colls/list.csv?datainfo\&rowcount\&interval=Silurian,Silurian\&cc=US\&e } \\
\text { nvtype=marine\&show=loc }\end{array}$ \\
\hline Devonian & $\begin{array}{l}\text { paleobiodb.org/data1.2/colls/list.csv?datainfo\&rowcount\&interval=Devonian,Devonian\&cc=US } \\
\text { \&envtype=marine\&show=loc }\end{array}$ \\
\hline Mississippian & $\begin{array}{l}\text { paleobiodb.org/data1.2/colls/list.csv?datainfo\&rowcount\&interval=Mississippian,Mississippian } \\
\text { \&cc=US\&envtype=marine\&show=loc }\end{array}$ \\
\hline Pennsylvanian & $\begin{array}{l}\text { paleobiodb.org/data1.2/colls/list.csv?datainfo\&rowcount\&interval=Pennsylvanian,Pennsylvania } \\
\text { n\&cc=US\&envtype=marine\&show=loc }\end{array}$ \\
\hline Permian & $\begin{array}{l}\text { paleobiodb.org/data1.2/colls/list.csv?datainfo\&rowcount\&interval=Permian,Permian\&cc=US\&e } \\
\text { nvtype=marine\&show=loc }\end{array}$ \\
\hline Triassic & $\begin{array}{l}\text { paleobiodb.org/data1.2/colls/list.csv?datainfo\&rowcount\&interval=Triassic,Triassic\&cc=US\&e } \\
\text { nvtype=marine\&show=loc }\end{array}$ \\
\hline Jurassic & $\begin{array}{l}\text { paleobiodb.org/data1.2/colls/list.csv?datainfo\&rowcount\&interval=Jurassic,Jurassic\&cc=US\&e } \\
\text { nvtype=marine\&show=loc }\end{array}$ \\
\hline E. Cretaceous & $\begin{array}{l}\text { paleobiodb.org/data1.2/colls/list.csv?datainfo\&rowcount\&interval=Early\%20Cretaceous,Early\% } \\
\text { 20Cretaceous\&cc=US\&envtype=marine\&show=loc }\end{array}$ \\
\hline L. Cretaceous & $\begin{array}{l}\text { paleobiodb.org/data1.2/colls/list.csv?datainfo\&rowcount\&interval=Late\%20Cretaceous,Late\%2 } \\
\text { 0Cretaceous\&cc=US\&envtype=marine\&show=loc }\end{array}$ \\
\hline Paleocene & $\begin{array}{l}\text { paleobiodb.org/data1.2/colls/list.csv?datainfo\&rowcount\&interval=Paleocene,Paleocene\&cc=U } \\
\text { S\&envtype=marine\&show=loc }\end{array}$ \\
\hline Eocene & $\begin{array}{l}\text { paleobiodb.org/data1.2/colls/list.csv?datainfo\&rowcount\&interval=Eocene,Eocene\&cc=US\&en } \\
\text { vtype=marine\&show=loc }\end{array}$ \\
\hline Oligocene & $\begin{array}{l}\text { paleobiodb.org/data1.2/colls/list.csv?datainfo\&rowcount\&interval=Oligocene,Oligocene\&cc=U } \\
\text { S\&envtype=marine\&show=loc }\end{array}$ \\
\hline Miocene & $\begin{array}{l}\text { paleobiodb.org/data1.2/colls/list.csv?datainfo\&rowcount\&interval=Miocene,Miocene\&cc=US\& } \\
\text { envtype=marine\&show=loc }\end{array}$ \\
\hline Pliocene & $\begin{array}{l}\text { paleobiodb.org/data1.2/colls/list.csv?datainfo\&rowcount\&interval=Pliocene,Pliocene\&cc=US\& } \\
\text { envtype=marine\&show=loc }\end{array}$ \\
\hline Pleistocene & $\begin{array}{l}\text { paleobiodb.org/data1.2/colls/list.csv?datainfo\&rowcount\&interval=Pleistocene,Pleistocene\&cc= } \\
\text { US\&envtype=marine\&show=loc }\end{array}$ \\
\hline Terrestrial & \\
\hline Devonian & $\begin{array}{l}\text { paleobiodb.org/data1.2/colls/list.csv?datainfo\&rowcount\&interval=Devonian,Devonian\&cc=US } \\
\text { \&envtype=terr\&show=loc }\end{array}$ \\
\hline Mississippian & $\begin{array}{l}\text { paleobiodb.org/data1.2/colls/list.csv?datainfo\&rowcount\&interval=Mississippian,Mississippian } \\
\text { \&cc=US\&envtype=terr\&show=loc }\end{array}$ \\
\hline Pennsylvanian & $\begin{array}{l}\text { paleobiodb.org/data1.2/colls/list.csv?datainfo\&rowcount\&interval=Pennsylvanian,Pennsylvania } \\
\text { n\&cc=US\&envtype=terr\&show=loc }\end{array}$ \\
\hline Permian & $\begin{array}{l}\text { paleobiodb.org/data1.2/colls/list.csv?datainfo\&rowcount\&interval=Permian,Permian\&cc=US\&e } \\
\text { nvtype=terr\&show=loc }\end{array}$ \\
\hline Triassic & $\begin{array}{l}\text { paleobiodb.org/data1.2/colls/list.csv?datainfo\&rowcount\&interval=Triassic,Triassic\&cc=US\&e } \\
\text { nvtype=terr\&show=loc }\end{array}$ \\
\hline
\end{tabular}




\begin{tabular}{|l|l|} 
Jurassic & $\begin{array}{l}\text { paleobiodb.org/data1.2/colls/list.csv?datainfo\&rowcount\&interval=Jurassic,Jurassic\&cc=US\&e } \\
\text { nvtype=terr\&show=loc }\end{array}$ \\
\hline L. Cretaceous & $\begin{array}{l}\text { paleobiodb.org/data1.2/colls/list.csv?datainfo\&rowcount\&interval=Early\%20Cretaceous,Early\% } \\
\text { 20Cretaceous\&cc=US\&envtype=terr\&show=loc }\end{array}$ \\
\hline U.. Cretaceous & $\begin{array}{l}\text { paleobiodb.org/data1.2/colls/list.csv?datainfo\&rowcount\&interval=Late\%20Cretaceous,Late\%2 } \\
\text { 0Cretaceous\&cc=US\&envtype=terr\&show=loc }\end{array}$ \\
\hline Paleocene & $\begin{array}{l}\text { paleobiodb.org/data1.2/colls/list.csv?datainfo\&rowcount\&interval=Paleocene,Paleocene\&cc=U } \\
\text { S\&envtype=terr\&show=loc }\end{array}$ \\
\hline Eocene & $\begin{array}{l}\text { paleobiodb.org/data1.2/colls/list.csv?datainfo\&rowcount\&interval=Eocene,Eocene\&cc=US\&en } \\
\text { vtype=terr\&show=loc }\end{array}$ \\
\hline Oligocene & $\begin{array}{l}\text { paleobiodb.org/data1.2/colls/list.csv?datainfo\&rowcount\&interval=Oligocene,Oligocene\&cc=U } \\
\text { S\&envtype=terr\&show=loc }\end{array}$ \\
\hline Miocene & $\begin{array}{l}\text { paleobiodb.org/data1.2/colls/list.csv?datainfo\&rowcount\&interval=Miocene,Miocene\&cc=US\& } \\
\text { envtype=terr\&show=loc }\end{array}$ \\
\hline Pliocene & $\begin{array}{l}\text { paleobiodb.org/data1.2/colls/list.csv?datainfo\&rowcount\&interval=Pliocene,Pliocene\&cc=US\& } \\
\text { envtype=terr\&show=loc }\end{array}$ \\
\hline Pleistocene & $\begin{array}{l}\text { paleobiodb.org/data1.2/colls/list.csv?datainfo\&rowcount\&interval=Pleistocene,Pleistocene\&cc= } \\
\text { US\&envtype=terr\&show=loc }\end{array}$ \\
\hline Note: These summarize the search parameters used for each download. All downloads were \\
made on Oct 24 -26, 2016.
\end{tabular}

\section{REFERENCES CITED}

Fortin, M.-J., 1999, Spatial statistics in landscape ecology, in Klopatek, C. C., and Gardner, R.

91 Haase, P., 1995, Spatial pattern analysis in ecology based on Ripley's K-function: Introduction and methods of edge correction: Journal of Vegetation Science, v. 6, no. 4, p. 575-582.

93 Heim, N. A., and Peters, S. E., 2011, Covariation in macrostratigraphic and macroevolutionary patterns in the marine record of North America: Geological Society of America Bulletin,

96 Plotnick, R. E., Gardner, R. H., Hargrove, W. W., Prestegaard, K., and Perlmutter, M., 1996, 
99 Plotnick, R. E., Gardner, R. H., and O'Neill, R. V., 1993, Lacunarity indexes as measures of 100 landscape texture: Landscape Ecology, v. 8, no. 3, p. 201-211.

101 Rosenberg, M. S., and Anderson, C. D., 2011, PASSaGE: Pattern Analysis, Spatial Statistics and 102 Geographic Exegesis. Version 2: Methods in Ecology and Evolution, v. 2, no. 3, p. 229232.

104 Young, L. J., and Young, J. H., 1998, Statistical Ecology, Boston, Kluwer Academic Publishers, 105 565 p.: 